\title{
Avaliação do potencial fisiológico de sementes de soja por meio de imagens ${ }^{1}$
}

\author{
Letícia Wendt $^{2}$, Francisco Guilhien Gomes Junior ${ }^{3}$, Maria de Fátima Zorato ${ }^{4}$, Gláucia Cristina Moreira ${ }^{5}$
}

\begin{abstract}
Evaluation of the physiological

potential of soybean seeds by image analysis

The use of seeds with a high physiological potential is very important for farmers, because it is directly related to high yield. This research aimed to evaluate the performance of soybean seeds (CD 2737 RR cultivar) from lots with high and low germination rates, as well as the efficiency of computerized analysis carried out by the Seed Vigor Imaging System (SVIS ${ }^{\circledR}$ ) and Tomato Analyzer softwares, in order to identify shape modifications in the harvested seeds and associate them with germination. Plant height, number of pods per plant, number of seeds per plant, water content, mass of 1,000 seeds and yield were evaluated. For vigor evaluation using the SVIS ${ }^{\circledR}$ software, the seedling length and indexes for growth, uniformity and vigor were determined. The seed morphology evaluation using the Tomato Analyzer software was carried out from the radiographic images of seeds. It was concluded that the highest seed yield and physiological potential were obtained from lots with high germination rates, when compared to the low germination ones, under greenhouse conditions. The SVIS ${ }^{\circledR}$ software showed to be efficient to identify vigor differences among lots, while the Tomato Analyzer, through the Fruit Shape Index External 2 and Circular parameters, was efficient to identify, in a fast and precise way, seeds with irregular shape associated with low germination.
\end{abstract}

KEY-WORDS: Glycine $\max ($ L.) Merrill; germination; X-ray.

\section{INTRODUÇÃO}

A soja é uma das mais importantes culturas agrícolas no Brasil, correspondendo a $40 \%$ do total de grãos produzidos. No mundo, a soja brasileira responde por $27 \%$ do mercado, e o País, além de ser

\section{RESUMO}

A utilização de sementes com alto potencial fisiológico é de grande importância para agricultores, pois está, diretamente, relacionada à alta produtividade. Este estudo objetivou avaliar o desempenho de sementes de soja (cultivar CD 2737 RR) produzidas a partir de lotes com alta e baixa germinação, bem como a eficiência da análise computadorizada, pelos programas Seed Vigor Imaging System (SVIS ${ }^{\circledR}$ ) e Tomato Analyzer, para identificar alterações no formato das sementes colhidas e associálas com a germinação. As variáveis analisadas foram: altura de planta, número de vagens por planta, número de sementes por planta, grau de umidade, massa de mil sementes e produtividade. $\mathrm{Na}$ avaliação do vigor, utilizando-se o programa $\mathrm{SVIS}^{\circledR}$, foram determinados o comprimento das plântulas e os índices de crescimento, uniformidade e vigor. A avaliação da morfologia das sementes pelo programa Tomato Analyzer foi realizada a partir de imagens radiográficas das sementes. Concluiu-se que a maior produtividade e o maior potencial fisiológico de sementes foram obtidos de lotes com alta germinação, em comparação com lotes de baixa germinação, em condições de casa-de-vegetação. O SVIS ${ }^{\circledR}$ mostrou-se eficiente para identificar diferenças de vigor entre os lotes e o Tomato Analyzer, por meio dos parâmetros Fruit Shape Index External 2 e Circular, para identificar, com rapidez e precisão, sementes com formato irregular, associado à baixa germinação.

PALAVRAS-CHAVE: Glycine $\max ($ L.) Merrill; germinação; raios $\mathrm{X}$.

o maior exportador, é, também, o maior produtor (Conab 2014). Sua importância é tão grande que, nos municípios em que o seu cultivo é mais intenso, o Índice de Desenvolvimento Humano (IDH) é superior ao das médias estadual e nacional. Estudos demonstram que os trabalhadores agrícolas empregados na

1. Trabalho recebido em ago./2013 e aceito para publicação em jul./2014 (nº registro: PAT 26141).

2. Universidade Estadual do Oeste do Paraná (Unioeste), Marechal Cândido Rondon, PR, Brasil. E-mail: wendtleticia@hotmail.com.

3. Universidade de São Paulo (USP), Escola Superior de Agricultura "Luiz de Queiroz", Departamento de Produção Vegetal,

Piracicaba, SP, Brasil.E-mail: francisco1@usp.br.

4. Grupo Aurora Seriös, Departamento de Controle de Qualidade de Sementes, Correntina, BA, Brasil.

E-mail: fatima.zorato@auroraserios.com.br.

5. Universidade Tecnológica Federal do Paraná (UTFPR), Departamento de Tecnologia de Alimentos, Medianeira, PR, Brasil. E-mail:gc_moreira@hotmail.com. 
cultura da soja são os mais bem remunerados, além de apresentarem grau de instrução mais elevado (Vernetti \& Vernetti Júnior 2009).

Ao longo dos anos, vem se buscando incrementar a produção de soja, no Brasil, por meio do aumento da área cultivada e da produtividade. Neste contexto, o uso de sementes de alta qualidade, na implantação de lavouras, assume papel fundamental (Schuch 2005).

A semente de soja, para ser considerada de alta qualidade, deve ter excelentes características fisiológicas e sanitárias, como altos níveis de vigor, germinação e sanidade, bem como garantia de pureza física e varietal. Estes fatores respondem pelo desempenho das sementes no campo, culminando com o estabelecimento da população de plantas requerida pela cultivar, aspecto fundamental que contribui para que sejam alcançados níveis altos de produtividade (França Neto et al. 2010).

$\mathrm{Na}$ avaliação do vigor de sementes de soja, vários testes têm sido recomendados, destacando-se os de envelhecimento acelerado, tetrazólio, condutividade elétrica, crescimento de plântulas e classificação do vigor de plântulas (Vieira et al. 2003). Entretanto, nos últimos anos, a pesquisa em tecnologia de sementes tem priorizado procedimentos computadorizados para a avaliação do vigor de sementes, como, por exemplo, a utilização do programa Seed Vigor Imaging System $\left(\right.$ SVIS $^{\circledR}$ ) (Sako et al. 2001, Hoffmaster et al. 2005). Na avaliação do vigor de sementes de soja, as análises SVIS ${ }^{\circledR}$ apresentam eficiência comparável à do teste de envelhecimento acelerado, constituindo alternativa interessante para a obtenção de informações consistentes sobre o potencial fisiológico das sementes (Marcos Filho et al. 2009).

As sementes de soja, devido às suas características morfológicas e químicas, destacam-se por serem bastante sensíveis à ação de fatores do ambiente (Marcos Filho 1979). A exposição das sementes em ciclos alternados de condições ambientais úmidas e secas, na fase de pós-maturidade, pode ocasionar danos por umidade, os quais apresentam maior magnitude quando ocorrem em ambientes quentes, típicos de regiões tropicais e subtropicais (Forti et al. 2010). A ocorrência de sementes de soja esverdeadas também tem sido associada a estresses bióticos e abióticos, interferindo, negativamente, na qualidade das sementes, com redução do potencial fisiológico e formação de sementes deformadas e alongadas (França Neto et al. 2012).
Nesse sentido, a identificação e separação de sementes com formatos irregulares tornam-se fundamentais para aprimorar a qualidade do lote de sementes. O programa Tomato Analyzer, desenvolvido para a avaliação de características morfológicas de frutos de tomate (Brewer et al. 2006) e, inicialmente, utilizado na pesquisa em sementes, para a avaliação de espaços livres internos à partir de imagens radiográficas (Marcos Filho et al. 2010), poderá constituir um procedimento rápido e eficiente na identificação do índice de anormalidades morfológicas, em lotes de sementes.

Assim, este trabalho objetivou avaliar o desempenho de sementes de soja produzidas em casa-de-vegetação, a partir de lotes com alta e baixa germinação, e avaliar o potencial fisiológico das sementes colhidas, utilizando-se os programas Seed Vigor Imaging System $\left(\mathrm{SVIS}^{\circledR}\right.$ ) e Tomato Analyzer, sendo o último para a identificação de alterações no formato das sementes, em associação com a germinação.

\section{MATERIAL E MÉTODOS}

O experimento foi realizado de abril a outubro de 2012, em casa-de-vegetação localizada na Cooperativa Central de Pesquisa Agrícola (Coodetec), situada na cidade de Cascavel (PR) (245 $57^{\prime} 20^{\prime \prime} \mathrm{S}$, $53^{\circ} 27^{\prime} 19^{\prime \prime} \mathrm{W}$ e altitude de $782 \mathrm{~m}$ ).

Para avaliar o efeito do potencial fisiológico das sementes de soja, cultivar CD 2737 RR (ciclo semiprecoce e crescimento indeterminado), foram utilizadas sementes provenientes de lotes com alta e baixa germinação: Lote 1: semente com alta germinação (96\%), produzida no Estado do Paraná; e Lote 2: semente com baixa germinação $(24 \%)$, produzida no Estado do Tocantins. O potencial fisiológico das sementes destes lotes foi determinado por meio de testes conduzidos em laboratório, descritos a seguir:

a) Teste de germinação: conduzido com quatro repetições de 50 sementes por lote, em rolos de papel toalha umedecidos com quantidade de água equivalente a 2,5 vezes a massa do papel seco, a $25^{\circ} \mathrm{C}$, sendo as plântulas avaliadas aos cinco dias após a semeadura (Brasil 2009). Os resultados foram expressos em percentagem média de plântulas normais para cada lote;

b) Teste de tetrazólio: utilizado para avaliar o vigor e a viabilidade das sementes (França Neto et al. 1998). Foram retiradas 100 sementes de cada amostra, pré-acondicionadas em papel de germinação umedecido com quantidade de água equivalente a 
2,5 vezes a sua massa, durante 24 horas, em temperatura ambiente. Em seguida, as sementes foram depositadas em copos plásticos de $50 \mathrm{ml}$, sendo adicionada solução na concentração de $0,075 \%$ de 2,3,5-trifenil-cloreto-de-tetrazólio, e colocadas no escuro, em estufa com temperatura de $40^{\circ} \mathrm{C}$, por três horas, sendo, posteriormente, lavadas em água corrente e analisadas individualmente;

c) Emergência de plântulas em areia: conduzida com duas repetições de 100 sementes para cada tratamento, semeadas em bandejas plásticas, à profundidade de $2 \mathrm{~cm}$, contendo, como substrato, areia esterilizada, umedecida a $60 \%$ da capacidade máxima de retenção de água do substrato (Brasil 2009). As bandejas foram mantidas em ambiente controlado, a $25^{\circ} \mathrm{C}$, durante cinco dias. Os resultados foram expressos em percentagem de plântulas normais.

As sementes foram submetidas a pré-germinação em rolo de papel, para selecionar as plântulas normais dos dois lotes, permanecendo em germinador a $25^{\circ} \mathrm{C}$, durante três dias. Em seguida, realizou-se transplantio de oito plântulas para vasos de polietileno de $10 \mathrm{~L}$, contendo substrato de terra esterelizado. Após 15 dias, realizou-se o raleio, permanecendo, nos vasos, apenas as três plantas mais bem desenvolvidas de cada lote, para avaliação da produtividade de cada amostra. O delineamento experimental utilizado foi em blocos casualizados, com 10 repetições para cada lote, contendo três plantas em cada vaso.

O experimento foi acompanhado semanalmente, observando-se a resposta de cada planta, em relação ao seu crescimento e desempenho. Após a colheita das plantas do experimento, foram realizadas as avaliações descritas a seguir, para os lotes 1' e 2' (provenientes dos lotes 1 e 2):

a) Altura de planta: determinada medindo-se cada planta do ápice da parte aérea até a superfície do substrato, com o auxílio de régua graduada em milímetros, expressando-se os valores em centímetros;

b) Número de vagens por planta: obtido pela separação e contagem do número total de vagens das três plantas de cada vaso, expressando-se o resultado médio do número de vagens por planta;

c) Massa de mil sementes: determinada utilizando-se oito subamostras de 100 sementes de cada lote, as quais foram pesadas em balança de precisão de 0,01 g (Brasil 2009), expressando-se os valores médios;

d) Número de sementes por planta: determinado pela contagem das sementes das 10 plantas de cada lote, expressando-se o resultado médio de sementes por planta;

e) Grau de umidade: determinado pelo método da estufa a $105 \pm 3^{\circ} \mathrm{C}$, por 24 horas, utilizando-se duas repetições para cada lote (Brasil 2009), com resultados expressos em percentagem na base úmida;

f) Produtividade: obtida pela colheita das plantas de cada vaso, que, após secas, foram trilhadas manualmente, pesadas e calculada a produtividade, com valores expressos em gramas por planta.

As sementes foram, também, submetidas a análises computadorizadas de imagens, no Laboratório de Análise de Imagens do Departamento de Produção Vegetal da Escola Superior de Agricultura "Luiz de Queiroz" (ESALQ) da Universidade de São Paulo (USP), em Piracicaba (SP).

Para a análise computadorizada de imagens de plântulas (SVIS ${ }^{\circledR}$ ), as plântulas foram obtidas de quatro repetições de 25 sementes para cada lote, colocadas para germinar em duas fileiras situadas no terço superior do papel toalha, a $25^{\circ} \mathrm{C}$, durante três dias. Após este período, as imagens foram captadas em escâner HP Scanjet G2410, disposto de maneira invertida, no interior de uma caixa de alumínio $(60,0 \mathrm{~cm} \times 50,0 \mathrm{~cm} \times 12,0 \mathrm{~cm})$, e operado pelo programa Photosmart $^{\mathbb{B}}$, com resolução de 100 dpi. As plântulas normais, anormais e sementes mortas identificadas em cada repetição foram transferidas do papel toalha para uma folha de cartolina de coloração preta, colocada sobre a plataforma interna da caixa metálica. As imagens digitalizadas foram analisadas pelo programa Seed Vigor Imaging System $\left(\mathrm{SVIS}^{\circledR}\right)$, sendo obtidos os valores médios do comprimento de plântulas, crescimento, uniformidade de desenvolvimento e índice de vigor, para cada lote (Sako et al. 2001). O índice de vigor foi proposto pela Ohio Seed Improvement Association, entidade certificadora de sementes no Estado de Ohio (EUA), que classifica plântulas com três dias de idade em vigor alto $(>500)$, médio (200-500) e baixo $(<200)$.

Para avaliação computadorizada de alterações morfológicas das sementes, quatro repetições de 25 sementes para cada lote foram radiografadas, utilizando-se equipamento digital da marca Faxitron X-Ray, modelo MX-20 DC-12, acoplado a um computador Core 2 Duo (3.16 GHz, 2 GB de memória RAM, Hard Disk de 160 GB e monitor MultiSync LCD1990SX de 17 polegadas). Após a obtenção das imagens radiográficas, as sementes foram colocadas em bandejas plásticas com células individualizadas 
(100 células por bandeja) e submetidas ao teste de germinação, conforme descrito anteriormente, sendo as sementes posicionadas no papel e mantendo-se a identificação inicial. A identificação de sementes com alterações morfológicas foi realizada pelo programa Tomato Analyzer (Brewer et al. 2006), utilizando-se os parâmetros Fruit Shape Index External 2, que expressa a relação entre o maior e o menor eixo delimitado na imagem radiográfica da semente, e Circular, que permite verificar o grau de aproximação da imagem radiográfica da semente com o formato circular delimitado pelo círculo inscrito. No terceiro dia após a germinação, foi realizada avaliação do comprimento individual das plântulas, por meio do SVIS $^{\circledR}$, sendo os resultados expressos em centímetros e relacionados com os valores dos parâmetros Fruit Shape Index External 2 e Circular de cada semente.

Para o procedimento estatístico, os dados foram submetidos à análise de variância, por meio do programa estatístico Assistat (Silva \& Azevedo 2002). Foi realizada análise de correlação linear entre a avaliação do comprimento de plântulas e os parâmetros do progama Tomato Analyzer. Os valores dos parâmetros Fruit Shape Index External 2 e Circular das sementes de cada lote foram apresentados na forma de gráficos elaborados pelo programa Microsoft Excel $^{\circledR}$, versão 14.0.

\section{RESULTADOS E DISCUSSÃO}

Foram verificadas diferenças visuais no desempenho das plântulas originadas dos dois lotes de sementes de soja, desde o início até o final do ciclo da cultura. As plântulas originadas de sementes com baixa germinação apresentaram atraso na maturação e colheita. Este comportamento foi evidenciado pelo aumento do período vegetativo, caracterizado pela presença de haste verde, na maioria das plantas.

Pelos resultados de germinação e de viabilidade obtidos no teste de tetrazólio, foram evidenciadas diferenças entre os lotes 1' e 2', produzidos a partir dos lotes 1 e 2 (Tabelas 1 e 2). Nas avaliações do teste de tetrazólio, foram observados altos percentuais de sementes com danos por umidade, sendo de $74 \%$ e $82 \%$, para os lotes 1' e 2', respectivamente. Estes resultados podem ser atribuídos ao estresse a que as plantas foram submetidas durante a fase final do ciclo de maturação, com a ocorrência de ciclos alternados de hidratação e secagem das sementes, devido à condensação da casa-de-vegetação.

As sementes colhidas apresentaram grau de umidade de $10,6 \%$ e $10,8 \%$ e massa de mil sementes de 171,9 g e 143,3 g, para os lotes 1'e 2', respectivamente. Na avaliação inicial, o lote 2 apresentou baixo potencial fisiológico (Tabelas 1 e 2), justificando a

Tabela 1. Teste de tetrazólio para avaliação do vigor e viabilidade de dois lotes de sementes de soja, cultivar CD 2737 RR (Cascavel, PR, 2012).

\begin{tabular}{|c|c|c|c|c|}
\hline \multirow{3}{*}{ Lote } & \multicolumn{2}{|c|}{ Sementes semeadas } & \multicolumn{2}{|c|}{ Sementes colhidas } \\
\hline & Vigor & Viabilidade & Vigor & Viabilidade \\
\hline & & & & \\
\hline 1 & 93 & 97 & 84 & 87 \\
\hline 2 & 30 & 67 & 62 & 65 \\
\hline F valor & $305,31 * *$ & $90,00^{*}$ & $24,20 *$ & $26,88^{*}$ \\
\hline C.V. $(\%)$ & 5,86 & 3,86 & 6,13 & 5,58 \\
\hline
\end{tabular}

* Significativo pelo teste $\mathrm{F}$, a $5 \%$; ** significativo pelo teste $\mathrm{F}$, a $1 \%$.

Tabela 2. Germinação de sementes e emergência de plântulas de soja em areia de dois lotes da cultivar CD 2737 RR (Cascavel, PR, 2012).

\begin{tabular}{|c|c|c|c|c|}
\hline \multirow{3}{*}{ Lote } & \multicolumn{2}{|c|}{ Sementes semeadas } & \multicolumn{2}{|c|}{ Sementes colhidas } \\
\hline & Germinação & Emergência & Germinação & Emergência \\
\hline & & & & \\
\hline 1 & 96 & 81 & 76 & 66 \\
\hline 2 & 24 & 11 & 54 & 37 \\
\hline F valor & $888,69 * *$ & $170,93 * *$ & $82,00 *$ & $45,46^{*}$ \\
\hline C.V. $(\%)$ & 5,74 & 16,46 & 5,21 & 8,27 \\
\hline
\end{tabular}


realização da pré-germinação, com o objetivo de selecionar plântulas normais e garantir o desenvolvimento das plantas até o final do ciclo, para os dois lotes de sementes (Tabela 2). Ressalta-se, entretanto, que as condições adversas de ambiente, ao final do ciclo das plantas, destacadas anteriormente, podem ter contribuído para os baixos valores de germinação e emergência de plântulas, para os lotes 1' e 2' (Tabela 2).

No teste de tetrazólio, o lote 2' apresentou vigor inferior ao do lote 1', confirmando os resultados obtidos no teste de emergência de plântulas em areia. Conforme Schuch (2005), as sementes vigorosas apresentam maior capacidade de transformação e suprimento de reservas dos tecidos de armazenamento e incorporação destas pelo eixo embrionário, afetando, desta forma, o processo de germinação e o crescimento das plântulas.

Com relação aos resultados da análise SVIS ${ }^{\circledR}$, foi observada sensibilidade equivalente aos resultados de germinação e emergência de plântulas. Os valores de comprimento de plântula, crescimento e índice de vigor foram maiores para o lote 1', com exceção do índice de uniformidade, que não permitiu diferir os lotes (Tabela 3).

Quanto ao índice de vigor, o lote 1' apresentou o valor de 565 , considerado alto para plântulas com três dias de idade, pela Ohio Seed Improvement Association. Estes resultados confirmam os dados apresentados na Tabela 2 e evidenciam a eficiência da análise computadorizada, conforme, também, observado por Marcos Filho et al. (2009), que concluíram que a análise de imagens de plântulas provenientes do teste de germinação ou do envelhecimento acelerado, com o uso do SVIS ${ }^{\circledR}$, é sensível para a determinação do vigor de sementes de soja.

As vantagens do $\mathrm{SVIS}^{\circledR}$, em comparação às avaliações realizadas com régua, incluem rápida obtenção de resultados e boa precisão, já que não envolvem erros humanos de avaliação, cálculo, estimativas e medições (Sako et al. 2001, Hoffmaster et al. 2005).

$\mathrm{Na}$ avaliação de alterações morfológicas das sementes colhidas, utilizando-se o programa Tomato Analyzer, foi observada, para os dois lotes, correlação negativa entre o comprimento de plântula e o parâmetro Fruit Shape Index External 2, com valores maiores e mais significativos para o lote 2' (Tabela 4). Quanto ao parâmetro Circular, foi observada correlação positiva deste com o comprimento de plântula apenas para as sementes do lote 2 '. Estes resultados indicam que sementes alongadas apresentam valores crescentes de Fruit Shape Index External 2, enquanto sementes com formato arredondado na imagem radiográfica, porém com deformações no tegumento, apresentam valores decrescentes para o parâmetro Circular.

De maneira geral, para uma mesma semente, quando o parâmetro Fruit Shape Index External 2 aumenta, o Circular diminui e mais irregular é o formato da semente. Invertendo-se o raciocínio, uma

Tabela 3. Resultados das avaliações SVIS ${ }^{\circledR}$ de dois lotes de sementes de soja, cv. CD 2737 RR, produzidas em casa-de-vegetação, a partir de sementes com alta e baixa germinação (lotes 1 e 2) (Cascavel, PR, 2012).

\begin{tabular}{lcccc}
\hline \multicolumn{1}{c}{ Lotes } & Comprimento de plântula $(\mathrm{cm})$ & Índice de crescimento & Índice de uniformidade & Índice de vigor \\
\hline $1^{\prime}$ & 4,4 & 472 & 785 & 565 \\
$2^{\prime}$ & 3,2 & 364 & 743 & 477 \\
\hline F valor & $29,53^{* *}$ & $18,40^{* *}$ & $0,96^{\text {ns }}$ & $26,04^{* *}$ \\
C.V. $(\%)$ & 8,56 & 8,48 & 7,94 & 4,67 \\
\hline
\end{tabular}

** Significativo pelo teste $\mathrm{F}$, a $1 \%$; ${ }^{\text {ns }}$ não significativo.

Tabela 4. Coeficientes de correlação linear (r) entre as avaliações de comprimento de plântula (CP) e os parâmetros Fruit Shape Index External 2 (FSIE) e Circular (CIR) do programa Tomato Analyzer utilizados para a avaliação da uniformidade de sementes de soja, cv. CD 2737 RR, produzidas em casa-de-vegetação, a partir de sementes com alta e baixa germinação (Cascavel, PR, 2012).

\begin{tabular}{lcccccc}
\hline & \multicolumn{3}{c}{ Lote 1 } & \multicolumn{2}{c}{ Lote 2' } \\
\cline { 2 - 6 } & CP & FSIE & CIR & CP & FSIE & CIR \\
\hline CP & - & $-0,2223^{*}$ & $0,1767^{\text {ns }}$ & - & $-0,4907^{* *}$ & $0,5287^{* *}$ \\
FSIE & - & - & $-0,8649^{* *}$ & - & - & $-0,9520^{* *}$ \\
\hline
\end{tabular}

* Significativo pelo teste $\mathrm{t}$, a $5 \%$; * significativo pelo teste $\mathrm{t}$, a $1 \%$; ${ }^{\text {ns }}$ não significativo. 
Tabela 5. Características agronômicas e produtividade de dois lotes de sementes de soja, cv. CD 2737 RR, produzidas em casa-devegetação, a partir de sementes com alta e baixa germinação (Cascavel, PR, 2012).

\begin{tabular}{|c|c|c|c|c|}
\hline Lotes & Altura da planta $(\mathrm{cm})$ & $\mathrm{N}^{\mathrm{o}}$ de vagens planta ${ }^{-1}$ & $\mathrm{~N}^{\circ}$ de sementes planta ${ }^{-1}$ & Produtividade $\left(\mathrm{g} \mathrm{planta}^{-1}\right)$ \\
\hline 1 & 157,8 & 32,5 & 76,3 & 13,1 \\
\hline 2 & 148,8 & 25,2 & 58,3 & 8,4 \\
\hline F valor & $3,82^{\mathrm{ns}}$ & $9,63 * *$ & $8,25^{*}$ & $25,80^{* *}$ \\
\hline C.V. $(\%)$ & 6,72 & 18,23 & 20,82 & 19,53 \\
\hline
\end{tabular}

* Significativo pelo teste $\mathrm{F}$, a $5 \%$;** significativo pelo teste $\mathrm{F}$, a $1 \%$; ${ }^{\text {ns }}$ não significativo.

semente de soja considerada dentro do padrão apresentaria valores crescentes de Circular e decrescentes de Fruit Shape Index External 2, ambos tendendo a 1,0 (Tabela 4). A utilização destes parâmetros do programa Tomato Analyzer apresenta-se como um procedimento rápido para a caracterização $\mathrm{e}$ identificação de sementes de soja fora do padrão de comercialização, visto que os resultados são obtidos em pouco tempo (menos de 1 minuto).

Sementes do lote 1' apresentaram valores de Fruit Shape Index External 2 inferiores a 1,4, enquanto, no lote 2', foram identificadas sementes com valores superiores a 1,6. Quanto ao parâmetro Circular, o lote 1' apresentou maior percentual de sementes com valores próximos de 1,0. Estes resultados indicam que as sementes provenientes do lote com menor potencial fisiológico apresentaram maior número de sementes deformadas. Uma das sementes analisadas, por apresentar valores Fruit Shape Index External 2 e Circular de 1,7488 e 0,8566, respectivamente, pode ser caracterizada como fora do padrão para comercialização. O resultado do teste de germinação evidenciou o baixo potencial fisiológico desta semente. Estes parâmetros podem ser úteis para o controle de qualidade de sementes em empresas, por avaliar, com rapidez, a uniformidade de lotes de sementes, com base em imagens de raios $\mathrm{X}$.

Com relação à produtividade e seus componentes (Tabela 5), verificou-se que o lote 1', com potencial fisiológico superior ao do lote 2' (Tabelas 1,2 e 3), apresentou maior número de vagens por planta, sementes por planta e produtividade, em relação ao lote 2'. Entretanto, para a avaliação da altura de planta, não foram observadas diferenças significativas entre os lotes de sementes.

Vanzolini \& Carvalho (2002), ao avaliarem o efeito do vigor de sementes de soja, observaram que este é maior no início do desenvolvimento das plantas, e que os lotes de menor vigor tiveram quedas mais acentuadas de estande. Houve atraso no início do florescimento, mostrando efeito do tipo residual no vigor das sementes.

Ao avaliarem o potencial fisiológico e a produtividade de sementes de soja, Scheeren et al. (2010) constataram que, para a altura de plantas, as sementes de alto vigor apresentaram resposta $11,5 \%$ superior à das sementes de baixo vigor, com diferença de $2,5 \mathrm{~cm}$ entre as plantas, a qual se manteve até os 75 dias após a semeadura. Isto permitiu que as plantas provenientes de sementes de alta qualidade tivessem uma vantagem desde o início, contribuindo para resultar em maior produtividade de sementes.

\section{CONCLUSÕES}

1. Maior produtividade e potencial fisiológico de sementes foram obtidos a partir de plântulas originadas de lotes com alta germinação, em comparação com lotes de baixa germinação, em casa-de-vegetação.

2. As avaliações SVIS $^{\circledR}$ de crescimento de plântulas e índice de vigor mostraram-se eficientes para identificar diferenças de vigor entre os lotes.

3. O programa Tomato Analyzer, por meio dos parâmetros Fruit Shape Index External 2 e Circular, mostrou-se eficiente para identificar, com rapidez e precisão, sementes com formato irregular, associado à baixa germinação.

\section{REFERÊNCIAS}

BRASIL. Ministério da Agricultura, Pecuária e Abastecimento. Secretaria de Defesa Agropecuária. Regras para análise de sementes. Brasília, DF: MAPA/ ACS, 2009.

BREWER, M. T. et al. Development of a controlled vocabulary and software application to analyze fruit shape variation in tomato and other plant species. Plant Physiology, Rockville, v. 141, n. 1, p. 15-25, 2006. 
COMPANHIA NACIONAL DE ABASTECIMENTO (Conab). Acompanhamento da safra brasileira de grãos. 2014. Disponível em: <www.conab.gov.br>. Acesso em: 04 abr. 2014.

FORTI, V. A.; CÍCERO, S. M.; PINTO, T. L. F. Avaliação da evolução de danos por "umidade" e redução do vigor em sementes de soja, cultivar TMG113-RR, durante o armazenamento, utilizando imagens de raios $\mathrm{X}$ e testes de potencial fisiológico. Revista Brasileira de Sementes, Londrina, v. 33, n. 3, p. 123-133, 2010.

FRANÇA NETO, J. B.; KRZYZANOWSKI, F. C.; COSTA, N. P. O teste de tetrazólio em sementes de soja. Londrina: Embrapa-CNPSo, 1998. (Documentos, 116).

FRANÇA NETO, J. B.; KRZYZANOWSKI, F. C.; HENNING, A. A. A importância do uso de sementes de soja de alta qualidade. Informativo Abrates, Londrina, v. 20, n. $1-2$, p. 37-38, 2010.

FRANÇA NETO, J. B. et al. Semente esverdeada de soja: causas e efeitos sobre o desempenho fisiológico série sementes. Londrina: Embrapa Soja, 2012. (Circular técnica, 91).

HOFFMASTER, A. F. et al. The Ohio State University Seed Vigor Imaging System $\left(\right.$ SVIS $^{\circledR}$ ) for soybean and corn seedlings. Seed Technology, Lansing, v. 27, n. 1, p. 7-24, 2005.

MARCOS FILHO, J. Qualidade fisiológica e maturação de sementes de soja (Glycine max (L.) Merrill). 1979. 180 f. Tese (Livre Docência) - Escola Superior da Agricultura “Luiz de Queiroz”, Universidade de São Paulo, Piracicaba, 1979.

MARCOS FILHO, J.; KIKUTI, A. L. P.; LIMA, L. B. de. Métodos para avaliação do vigor de sementes de soja, incluindo a análise computadorizada de imagens. Revista Brasileira de Sementes, Londrina, v. 31, n. 1, p. 102-112, 2009.

MARCOS FILHO, J. et al. Using tomato analyzer software to determine embryo size in X-rayed seeds. Revista Brasileira de Sementes, Londrina, v. 32, n. 2, p. 146-153, 2010.

SAKO, Y. et al. A system for automated seed vigour assessment. Seed Science and Technology, Zürich, v. 29, n. 3, p. 625-636, 2001.

SCHEEREN, B. R. et al. Qualidade fisiológica e produtividade de sementes de soja. Revista Brasileira de Sementes, Londrina, v. 32, n. 3, p. 35-41, 2010.

$\mathrm{SCHUCH}$, L. O. B. Maximizando a produção com sementes de alto vigor. SEED News, Pelotas, v. 10, n. 3, p. 8-11, 2005 .
SILVA, F. A. S.; AZEVEDO, C. A. V. Versão do programa computacional Assistat para o sistema operacional Windows. Revista Brasileira de Produtos Agroindustriais, Campina Grande, v. 4, n. 1, p. 71-78, 2002.

VANZOLINI, S.; CARVALHO, N. M. Efeito do vigor de sementes de soja sobre o seu desempenho em campo. Revista Brasileira de Sementes, Londrina, v. 24, n. 1, p. 33-41, 2002.

VERNETTI, F. de J.; VERNETTI JÚNIOR, F. de J. V. Genética da soja: caracteres qualitativos e diversidade genética. Brasília, DF: Embrapa Informação Tecnológica, 2009.

VIEIRA, R. D.; BITTENCOURT, S. R. M.; PANOBIANCO, $M$. Seed vigour: an important component of seed quality in Brazil. ISTA - Seed Testing International, Zürich, v. 126, n. 1, p. 21-22, 2003. 